Voix et Images

\title{
BIBLIOGRAPHIE DU CORPUS THÉORIQUE SUR LA BANDE DESSINÉE QUÉBÉCOISE
}

\section{JEAN-MICHEL BERTHIAUME}

Volume 43, numéro 2 (128), hiver 2018

La bande dessinée québécoise

URI : https://id.erudit.org/iderudit/1045068ar

DOI : https://doi.org/10.7202/1045068ar

Aller au sommaire du numéro

Éditeur(s)

Université du Québec à Montréal

ISSN

0318-9201 (imprimé)

1705-933X (numérique)

Découvrir la revue

Citer ce document

BERTHIAUME, J.-M. (2018). BIBLIOGRAPHIE DU CORPUS THÉORIQUE SUR LA BANDE DESSINÉE QUÉBÉCOISE. Voix et Images, 43(2), 113-124.

https://doi.org/10.7202/1045068ar d'utilisation que vous pouvez consulter en ligne.

https://apropos.erudit.org/fr/usagers/politique-dutilisation/ 


\title{
B IBLIOGR A PHIE D U CORPUS THÉORIQUE SUR LA BANDE DESSINÉE QUÉBÉCOISE ${ }^{1}$
}

\author{
$+++$ \\ JEAN-MICHEL BERTHIAUME \\ Université du Québec à Montréal
}

\section{M O N O G R A P H I E S}

+ AIRD, Robert et Mira FALARDEAU, Histoire de la caricature au Québec, Montréal, VLB éditeur/ Chaire Hector-Fabre d'histoire du Québec, coll. «Études québécoises», 2009, 248 p.

+ ALLARD, Yvon, Les bandes dessinées pour adultes, Montréal, Librairie Demarc, 1983, 6 p.

+ ARSENEAU-BUSSIÈRES, Philippe, Imagine ta B.D., Lévis, Éditions À reproduire, 1994, $50 \mathrm{f}$.

+ BEAUliEU, Jimmy et David TURGEON, Jimmy Beaulieu: L'œil amoureux. Entretiens avec David Turgeon au sujet de Comédie sentimentale pornographique, [s. 1.], Colosse, coll. «Nouvelle collection Colosse», [s. d.], 44 p.

+ BEAUliEU, Jimmy, Rôles en coulisses, Montréal, Jimmy Beaulieu éditeur, 2016, 44 p.

+ BELL, John (dir.), Canuck Comics: a Guide to Comic Books Published in Canada, avec la collaboration de Luc Pomerleau et Robert MacMillan, Montréal/Buffalo, Matrix Books/ University of Toronto Press, 1986, 154 p.

+ BERNARD, Lise, Bandes dessinées, illustrations de Daniel Dumont et de Jacques Goldstyn, Boucherville, Graficor, coll. «Les Scribouillards», 1988, 32 p.

+ BLETON, Paul, De Bécassine à Délirius. Bande dessinée et figuration narrative, illustrations de Christian-Marie Pons, Sainte-Foy, Télé-Université, 1992, 302 p.

+ BROWN, Andy (dir.), BDQ. Essays and Interviews on Quebec Comics, Greenwich, Conundrum Press, 2017, $224 \mathrm{p}$.

+ CAMBRON, Micheline et Dominic HARDY, Quand la caricature sort du journal. Baptiste Ladébauche, 1878-1957, avec la collaboration de Nancy Perron, Fides, Montréal, 2015, 323 p.

+ CARPENTIER, André (dir.), La bande dessinée kébécoise, Bois-des-Filion, La Barre du Jour, $1975,260 \mathrm{p}$.

+ CARPENTIER, André, Journal de mille jours. Carnets 1983-1986, Montréal, Guérin littérature/ XYZ éditeur, 1988, 354 p.

1 Cette bibliographie est non exhaustive. Néanmoins, nous avons tenté d'y inclure un important échantillonnage de textes critiques provenant d'une grande variété de sources. L'histoire de la bande dessinée reste vivante notamment grâce à des initiatives citoyennes, et nous avons décidé de prendre en compte cet aspect lors de la rédaction de cette bibliographie. De plus, nous ne pouvons passer sous silence l'aide inestimable de Michel Viau qui, durant des années, a épluché les périodiques québécois des quatre coins de la province pour conserver les traces des articles écrits sur cet art. Nous voulons aussi remercier Jean-Dominic Leduc, Sylvain Lemay et Gabriel Tremblay-Gaudette de leur expertise dans l'élaboration de cette bibliographie. 
+ COLleCtif, La Pastèque, 15 ans d'édition, Montréal, Éditions de la Pastèque, 2013, 270 p.

+ CÔTÉ, André-Philippe et Gilles PERRON, De la caricature et de la bande dessinée, Paroisse Notre-Dame-des-Neiges, Éditions Trois-Pistoles, coll. «Écrire», 2003, 121 p.

+ CÔTÉ, André-Philippe et Mira FALARDEAU, L'oreille coupée et autres scénarios, scénarios illustrés par les auteurs et par Jean-François Bergeron [Djief], Québec, L'Instant même, coll. «L'instant ciné», 2007, 115 p.

+ DUBOIS, Bernard, Bande dessinée québécoise. Répertoire bibliographique à suivre, Sillery, Éditions D.B.K., 1996, 105 p.

+ FALARDEAU, Mira, L'humour visuel: histoire et technique. Caricature, bande dessinée, dessin animé, Québec, École des arts visuels/Université Laval, coll. «Cahiers de communication graphique», 1976, $75 \mathrm{f}$.

+ FALARDEAU, Mira, La bande dessinée au Québec, Montréal, Boréal, coll. «Boréal express», 1994, $125 \mathrm{p}$.

+ FALARDEAU, Mira, Histoire de la bande dessinée au Québec, Montréal, VLB éditeur, coll. «Études québécoises», 2008, 190 p.

+ FRÉMION, Yves, Le guide de la bédé francophone, avec des photos de Christine Poutout, Paris, Syros alternatives, coll. "Les guides culturels Syros», 1990, 191 p.

+ HIRSH, Michael et Patrick LOUBERT, The Great Canadian Comic Books, Toronto, Peter Martin Associates, 1971, $264 \mathrm{p}$.

+ HONOREZ, Amélie, La représentation du genre féminin dans la bande dessinée québécoise. Étude de cas: Les nombrils, Paris, Presses académiques francophones, coll. «Omn. Pres. Franc.», 2014, 108 p.

+ HUDON, François, Normand Hudon, 1929-1997. Une vie comme un arc-en-ciel de couleurs, Montréal, Lidec, coll. «Célébrités», 2001, 61 p.

+ LANGLOIS, Richard, Bande dessinée et figuration narrative : textes et documents, Sherbrooke, Collège de Sherbrooke, Département de français, 4e édition, 1989, 300 p.

+ LEDUC, Jean-Dominic, Demi-dieux. 40 ans de super-héros de la bande dessinée québécoise, Montréal, Mém9ire, 2013, 157 p.

+ LEDUC, Jean-Dominic et Michel VIAU, Les années Croc. L'histoire du magazine qu'on riait, Montréal, Québec Amérique, 2013, 415 p.

+ LEMAY, Sylvain, Du Chiendent dans le printemps. Une saison dans la bande dessinée québécoise, Montréal, Mém9ire, 2016, 157 p.

+ NADEAU, Jean-François, La Palme: la caricature et autres sujets sérieux, Montréal, L'Hexagone, coll. «Entretiens», 1997, $151 \mathrm{p}$.

+ ROBIDOUX, Léon A., Albéric Bourgeois, caricaturiste, préface de Normand Hudon et Robert LaPalme, Montréal, VLB éditeur/Médiabec, 1978, 290 p.

+ SAMSON, Jacques et André CARPENTIER, Actes. Premier Colloque de bande dessinée de Montréal, Montréal, Analogon, 1986, 223 p.

+ TESSIER, Marc, Bédéistes en territoire québécois: 30 ans d'images, entrevue de Jean-Dominic LEDUC et postface de Jacques SAMSON, Québec, Moelle graphique, 2015, 102 p.

+ TURGEON, David, Le magnétophone de Yoko et autres essais, Vincent Giard éditeur, coll. "Collosse. Essais», 2014, 123 p.

+ VIAU, Michel, BDQ : répertoire des publications de bandes dessinées au Québec. Des origines à nos jours, Laval, Éditions Mille-Îles, coll. «Argus», 1999, 342 p.

+ VIAU, Michel, BDQ : histoire de la bande dessinée au Québec, t. I: Des origines à 1979, Montréal, Mém9ire, 2014, 343 p. 


\section{I. A R T I C L E S}

+ AL + FLAG \& LANG, «Café Noir et sans sucre artificiel», Trip, n 7, printemps 2012, p. 149-155.

+ APOSTOLIDẼS, Jean-Marie, «Les rituels de Luc Giard», Formule, n 1, 2007, p. 52-79.

+ ARCAND, Bernard et Sylvie VINCENT, «Il y a un dessein dans le Boréal Express», Recherches amérindiennes au Québec, vol. VIII, nº 3, 1979, p. 203-212.

+ BADO [Guy Badeau], «De la BDK à la BDQ», Liaison, n 108, septembre 2000, p. 20.

+ BEAULIEU, Jimmy, «Ce pont au sourire crâneur» [préface], Luc Giard, Le pont du Havre, Montréal, Mécanique générale/Les 400 coups, 2005, p. 4-5.

+ BEAUlIEU, Jimmy, "Automythology», European Comic Art, vol. V, nº 1, printemps 2012, p. 25-56.

+ BERTHIAUME, Jean-Michel, «Marc Tessier, le magicien», Sentinelle, n 1, avril 2015, p. 55-61.

+ BERTHIAUME, Jean-Michel, «Entrevue avec Zviane», Trip, n 8, printemps 2014, p.157-163.

+ BLANCHARD, Gérard, «Les bandes dessinées québécoises», Communication et langages, nº 19, 1973, p. 47-62.

+ BOULÉ, Jean-François, "Quelques élucubrations et autres troubles diégétiques de la bande dessinée québécoise», Trip, nº 7, printemps 2012, p. 87-88.

+ BORDELEAU, Francine, "La revanche des bédéistes québécois», Lettres québécoises. La revue de l'actualité littéraire, no 118, été 2005, p. 13-16.

+ BOUCHARD, Éric, "L'ostie d'chat, la bande dessinée en colocation», Trip, nº 7, printemps 2012 , p. 127-134.

+ BOUCHARD, Éric, "La suggestion sensorielle dans Down in the patawawa», Trip, $\mathrm{n}^{\circ}$ 8, printemps 2014, p. 143-147.

+ BOUCHARD, Éric, «L'homme à tête d'ours ou Comment devenir conteur? ", Trip, n 8, printemps 2014, p. 193-197.

+ BOUCHARD, Éric, «Relectures: Susceptible de Geneviève Castrée», Planches, n 7, printemps 2016, p. 39-42.

+ BOUCHARD, Éric, «Relectures: Kaspar de Obom», Planches, n 8, automne 2016, p. 87-90.

+ BOUCHARD, Éric, "Relectures: Du chez-soi d’Ariane Dénommé», Planches, nº 9, hiver 2016, p. 92-96.

+ BOUCHARD, Éric, «Relectures: Vers les mondes lointains de Grégoire Bouchard», Planches, $\mathrm{n}^{\circ}$ 10, printemps 2017, p. 31-34.

+ BOUCHARD, Éric, «Relectures: Jimmy et le Bigfoot de Pascal Girard», Planches, n 12, automne 2017, p. 63-66.

+ BOUCHER, Nicolas, «Les strips au Québec», Trip, n 7, printemps 2012, p. 156-159.

+ BRIND'AMOUR, Serge, "Zap! Pow! Stie! La nationalisation de la bande dessinée», Maclean's, vol. XIV, nº 7, juillet 1974, p. 17-21 et 45-46.

+ CANTIN, Pierre, «La bande dessinée québécoise», René Dionne (dir.), Le Québécois et sa littérature, Sherbrooke/Paris, Éditions Naaman/Agence de coopération culturelle et technique, coll. «Littératures», 1984, p. 398-406.

+ CARPENTIER, André, «La parole à Chiendent», La Barre du Jour, n ${ }^{\text {os }} 46-47-48-49$, $1^{\text {er }}$ trimestre 1975, p. 53-56.

+ CARPENTIER, André, «La bande dessinée québécoise passe au Salon...», Perspectives, 24 janvier 1976, p. 10-12.

+ CAUCHON, Paul, «La bande dessinée québécoise: une adolescence prolongée», Imagine..., $n^{\circ} 28$, juin 1985, p. 24-30. 
+ CHARBONNEAU-GRENIER, Laurent-Anthony, «La bande dessinée numérique: un cas particulier», Québec français, nº 168, hiver 2013, p. 32-34.

+ CHAREST-SIGOUIN, Violaine, «François Lapierre: d'ombres et de couleurs», Entre les lignes, vol. IV, nº 3, printemps 2008, p. 33.

+ CLICHE, Mira, «Éclosion d'un art», Entre les lignes, vol. IV, n 3, printemps 2008, p. 19-24.

+ DAGENAIS, Émilie, «Les dessous de la création: Cathon et Alexandre Fontaine Rousseau dans les coulisses des Cousines Vampires ", Planches, n 1, automne 2014, p. 35-40.

+ DAGENAIS, Émilie, «Les dessous de la création: Yan Mongrain nous parle de Dominique», Planches, $\mathrm{n}^{\circ}$ 2, hiver 2015, p. 25-30.

+ DAGENAIS, Émilie, «Les dessous de la création: Domaine Grisloire avec Michel Falardeau», Planches, $n^{\circ}$ 3, printemps 2015, p.19-26.

+ DAGENAIS, Émilie, «Les dessous de la création: Ping-Pong avec Zviane», Planches, nº 4, été 2015, p. 29-36.

+ DAGENAIS, Émilie, «Les dessous de la création: Namesake, Isabelle Melançon et Megan LaveyHeaton", Planches, nº 5, automne 2015, p. 24-28.

+ DAGILIS, Andrew, «Julie Doucet's Secretions», The Comics Journal, no 141, avril 1991, p. 98-105.

+ DANAUX, Stéphanie, «Une étape méconnue de l'humour graphique: les bandes dessinées de La Presse et de La Patrie, 1904-1910. Perspectives de recherche pour l'histoire de l'art», Globe. Revue internationale d'études québécoises, vol. XV, nos 1-2, 2012, p. 129-159.

+ DARDAILlON, Sylvie et Christophe MEUNIER, «La série Paul de Michel Rabagliati: récits d'espaces et de temps», Comicalités, avril 2013, en ligne: https://journals.openedition.org/comi calites/1566?lang=en\&gathStatIcon=true (page consultée le 8 février 2018).

+ DELPORTE, Julie, «Les images mouvementées de Vincent Giard», du9, avril 2009, en ligne: https:// www.du9.org/dossier/images-mouvementees-de-vincent/(page consultée le 20 janvier 2018).

+ DELPORTE, Julie, «Entretien avec David Turgeon», Trip, n 7, printemps 2012, p. 190-191.

+ DEMERS, Pierre, «Paulin Lessard, pionnier oublié de la B.D. québécoise», Antennes, ns 15-16, 1979, p. 14-18.

+ ÉMOND-FERRAT, Jessica, «Blogue à part», Entre les lignes, vol. IV, n 3, printemps 2008, p. 32.

+ FALARDEAU, Mira, "Les bédessinatrices», La Nouvelle Barre du Jour, nºs 110-111, février 1982, p. 81-87.

+ FALARDEAU, Mira, «La BD française est née au Canada en 1904 », Communication et langages, no 126, décembre 2000, p. 23-46.

+ FALARDEAU, Mira, «Figure de l'inversion dans la bande dessinée féministe», Trip, nº 7 , printemps 2012, p. 201-209.

+ FAUCHER, Christine, «Une entrevue avec Nicolas Plamondon», Vision, n 64, novembre 2006, p. 10-14.

+ FONTAINE ROUSSEAU, Alexandre, «Représentation du cinéma québécois dans la bande dessinée québécoise», Trip, nº 7, printemps 2012, p. 121-123.

+ FONTAINE ROUSSEAU, Alexandre, «Pour une politique de l'espace», Sentinelle, n 1, avril 2015, p. 51-53.

+ FONTAINE ROUSSEAU, Alexandre, «Les dessous de la création: Catherine Ocelot: Dans les coulisses de Talk-Show», Planches, n 7, printemps 2016, p. 11-16.

+ FONTAINE ROUSSEAU, Alexandre, «Les dessous de la création: Michel Hellman, dans les coulisses de Nunavik», Planches, n 8, automne 2016, p. 44-49. 
+ FONTAINE ROUSSEAU, Alexandre, «Les dessous de la création: dans les coulisses d'Hiver Nucléaire 2 avec Cab», Planches, n 9, hiver 2016, p. 55-61.

+ FONTAINE ROUSSEAU, Alexandre, «Les dessous de la création: dans les coulisses des 500 premiers Cadieux avec Xavier Cadieux», Planches, n 10, printemps 2017, p. 78-82.

+ FONTAINE ROUSSEAU, Alexandre, «Les dessous de la création: Boum dans les coulisses de Boumeries", Planches, ${ }^{\circ}$ 13, hiver 2017, p. 33-37.

+ FORTIER, Yann, «Les aventures de la bédé québécoise», Forces, vol. XLIII, nº 160, hiver 20092010, p. 48-54.

+ FRÉRET, Nicolas, «Le grand défi du Prix de la critique de la bande dessinée québécoise», Sentinelle, n 2, août 2015, p. 83-91.

+ GAGLIARDI, Laurent et Pierre GUÉNETTE, «Raoul Barré (1874-1932). Notes biographiques», Association des illustrateurs et illustratrices du Québec, communiqué n 5, avril 1985, p. 6-8.

+ GIARD, Vincent, «Les dessous de la création: Luc Bossé: B.D.G.», Planches, n 6, hiver 2016, p. 39-45.

+ GIAUFRET, Anna, «La BD québécoise. Magasin général, la langue entre imaginaire et représentation", Publif@rum, nº 14, 2011, en ligne: http://publifarum.farum.it/ezine_articles. php?art_id=184 (page consultée le 20 janvier 2018).

+ GIAUFRET, Anna, «Le français dans la bande dessinée québécoise: quelles représentations du français parlé?», Repères DoRiF, n² 2, volet 2, juillet 2013, en ligne: http://www.dorif.it/ezine/ ezine_articles.php?art_id=74 (page consultée le 20 janvier 2018).

+ GIAUFRET, Anna, «Normes et représentations du français québécois dans la bande dessinée québécoise des jeunes auteurs montréalais», Sentinelle, nº 3, décembre 2015, p. 17-29.

+ GIAUFRET, Anna, "La représentation du français québécois parlé dans les bandes dessinées des jeunes auteurs montréalais francophones. Une étude de corpus», Cahiers internationaux de sociolinguistique, $\mathrm{n}^{\circ}$ 10, août 2016, p. 205-232.

+ GIGUÈRE, Michel, «Michel Rabagliati. La vie vaut la peine d'être dessinée», leslibraires.ca, $1^{\text {er }}$ septembre 2006, en ligne: https://revue.leslibraires ca/entrevues/bande-dessinee/michelrabagliati-la-vie-vaut-la-peine-detre-dessinee (page consultée le 20 janvier 2018).

+ GODIN-LAVERDIĖRE, Julie-Anne et Myriam BARRIAULT-FORTIN, «Parodier et satiriser l'histoire par la bande dessinée, la caricature et la chronique humoristique. Le cas d'Albéric Bourgeois (1876-1962)», Recherches sociographiques, vol. LVI, nº 1, janvier-avril 2015, p. $53-83$.

+ GRAYSON-C., Catherine-Lune, "Grand cru. Les bédéistes Sylvie Rancourt et Jacques Boivin", Montréal Campus, 8 mars 2000, p. 14.

+ HARDY, Dominic, "Henri Julien, "ce diable d'homme" », Micheline Cambron (dir.), La vie culturelle à Montréal vers 1900, Montréal, Fides/Bibliothèque nationale du Québec, 2005, p. $157-173$.

+ HÉBERT, François, «Hérauts, première véritable revue de bandes dessinées québécoises », La Nouvelle Barre du Jour, $\mathrm{n}^{\text {os }}$ 110-111, février 1982, p. 113-120.

+ HURTUBISE, Jacques, «La bande dessinée québécoise», Québec français, nº 26, mai 1977, p. 41-45.

+ HURTUBISE, Jacques, «Petite bibliothèque de base de la bande dessinée», Québec français, $n^{\circ} 26$, mai 1977, p. 48-55.

+ HURTUBISE, Jacques, «Comment ou pourquoi on devient créateur de bande dessinée au Québec», La Nouvelle Barre du Jour, nos 110-111, février 1982, p. 123-128. 
+ JETTÉ, Marc, «Veena, d'un univers de papier à la réalité», Trip, no 7, printemps 2012, p. 63-65.

+ JETTÉ, Marc, «Bernie Mireault, une œuvre à découvrir», Trip, nº 8, printemps 2014, p. 177-183.

+ LA PALME, Robert, «La caricature: des figurines antiques au cartoon québécois», Vie des arts, nº67, été 1972, p. 11-18.

+ LACROIX, Saël, «André Montpetit, un oublié de la nuit», Sentinelle, n 3, décembre 2015, p. 7-14.

+ LACROIX, Yves, «La bande dessinée dans les journaux québécois (1930-1950). Un inventaire», La Nouvelle Barre du Jour, nos 110-111, février 1982, p. 101-109.

+ LAMOTHE, Stéphanie, Petite histoire de l'autobiographie dans la BD québécoise, pop-en-stock.ca, 3 mai 2012, en ligne: http://popenstock.ca/petite-histoire-de-lautobiographie-dans-la-bdquebecoise (page consultée le 20 janvier 2018).

+ LANGLOIS, Richard, «Sherbrooke, berceau et centre de la bande dessinée québécoise», L'album, Sherbrooke, BD Estrie, 1988, p. 36-38.

+ LAPLANTE, Robert, «Les comics Héritage. Des madeleines bien musclées», Sentinelle, nº 2, août 2015, p. 71-81.

+ LAPORTE, Louise, "Les souvenirs du pionnier de la bande dessinée: Albert Chartier», Ensemble. Le magazine de la vie à deux, vol. I, nº 5, janvier 1979, p. 8-9.

+ LEDUC, Jean-Dominic, «Jocelyn Houde, l'illustre inconnu», Sentinelle, nº 1, avril 2015, p. 33-39.

+ LEDUC, Jean-Dominic, "Bernie Mireault, un canadien errant», Sentinelle, n 3, décembre 2015, p. 31-45.

+ LEMAY, Sylvain, «Le Chiendent de la bande dessinée au Québec», Littératures, n²0, 1999, p. 133-147.

+ LEMAY, Sylvain, «Les tentatives de constitution d'un champ de la bande dessinée au Québec», Les cahiers du CÉDEL, n 3, 1999, p. 67-76.

+ LEMAY, Sylvain, «Panorama de la bande dessinée québécoise pour la jeunesse (1970-2000)», Françoise Lepage (dir.), La littérature pour la jeunesse, 1970-2000, Montréal, Fides, coll. "Archives des lettres canadiennes», 2003, p. 99-117.

+ LEMAY, Sylvain, "L'hommage trop discret à Albert Chartier», Le Devoir, $1^{\text {er }}$ mars 2004, p. A6.

+ LEMAY, Sylvain, «Les aventures de la bande dessinée en Outaouais», Au fait, vol. XI, n 3 , 2005, p. 3-4.

+ LEMAY, Sylvain, «L'appel de la race ou l'influence des livres. L'exemple des Contes historiques de la Société Saint-Jean-Baptiste de Montréal (1919-1925)», Formule, nº 1, 2007, p. 80-94.

+ LEMAY, Sylvain, «Pour une cinquième saison de la bande dessinée québécoise», Trip, nº 6, septembre 2009, p. 19-30.

+ LEMAY, Sylvain, «Michel Risque: le sémiologue analpha-bête», Trip, n 7, printemps 2012, p. $12-28$.

+ LEMAY, Sylvain, «BD québécoise: l'explosion», À bâbord!, n 41, octobre-novembre 2011, en ligne: https://www.ababord.org/BD-quebecoise-l-explosion (page consultée le 20 janvier 2018).

+ LEMAY, Sylvain, «Red Ketchup, pour une sémiologie appliquée», Trip, n 8, printemps 2014, p. 165-174.

+ LEMAY, Sylvain et Jean-François BOULÉ, «Le personnage féminin dans les bandes de L'Action catholique (1935-1938)", Alternative francophone, vol. I, n 9, 2016, p. 93-107, en ligne: https://journals. library.ualberta.ca/af/index.php/af/article/view/25711 (page consultée le 20 janvier 2018). 
+ LETOVSKY, Cliff, «Komiks Kebek», Le Beaver, ns 23-25, juin-août 1973, p. 87-93.

+ LORD, Denis, "Chu malade kalisse! Le corps moderne chez Henriette Valium», Esse arts + opinions, $\mathrm{n}^{\circ}$ 29, juillet 1996.

+ LORD, Denis, «Bulles de lys (et phylactères de nos aïeux)», Esse arts + opinions, n 39, printemps-été 2000, p. 68-74.

+ LORD, Denis, «Victor et Rivière, les stratégies esthétiques», Trip, nº 7, printemps 2012, p. 73-75.

+ MACLEOD, Catriona, «Sex and Death in Quebec: Female AutobioBD and Julie Doucet's Changements d'adresses ", European Comic Art, vol. V, n 1, printemps 2012, p. 57-70.

+ MARSHALL, Bill, «European Comic Art and Quebec», European Comic Art, vol. V, n 1 , printemps 2012, p. 4-8.

+ MARTIN, Annabelle, «Luis Neves, à la recherche de Quinquin», Trip, nº 7, mars 2010, p. 141-145.

+ MAYEUX, François (dir.), dossier «La bande dessinée en ébullition», Entre les lignes, vol. IV, $\mathrm{n}^{\circ}$ 3, printemps 2008, p. 18-35.

+ MCLEOD, Bob, «Featured Artist: Yanick Paquette», Rough Stuff, n 3, hiver 2007, p. 29-37.

+ MELANÇON, Benoît, "Les fanzines québécois», Revue d'histoire littéraire du Québec et du Canada français, nº 6, été-automne 1983, p. 95-98.

+ MELANÇON, Benoît, "BDHQ: bande dessinée et hockey au Québec», Benoît Melançon et Michel Porret (dir.), Pucks en stock. Bande dessinée et sport, Chêne-Bourg [Suisse], Georg éditeur, coll. «L'Équinoxe», 2016, p. 101-117.

+ MEUNE, Manuel, «De la Guerre froide à la guerre des langues: Tintin au pays de la traduction. Les adaptations en langues régionales dans l'espace francophone», Viviane Alary et Benoît Mitaine (dir.), Lignes de front. Bande dessinée et totalitarisme, Chêne-Bourg [Suisse], Georg éditeur/Colloque de Cerisy, coll. «L'Équinoxe», 2011, p. 165-182.

+ MILLER, Ann et Murray PRATT, «Transgressive Bodies in the Work of Julie Doucet, Fabrice Neaud and Jean-Christophe Menu. Towards a Theory of the "AutobioBD"», Belphégor. Littérature populaire et culture médiatique, vol. IV, n 1, novembre 2004, en ligne: http:// dalspace.library.dal.ca/bitstream/handle/10222/47696/04_01_Miller_trnsgr_en_cont.pdf? sequence=1\&isAllowed=y (page consultée le 20 janvier 2018).

+ MILLET, Yves, «Ā Montréal, en B.D., qu'y a-t-il de montréalais?», Esse arts + opinions, nº 10, printemps-été 1988, p. 47-51.

+ MILLET, Yves, «Luis Neves, quelques réflexions sur l'œuvre», Trip, nº 7, printemps 2012, p. 147.

+ MONTPETIT, Charles, "C'est si dur de dessiner des dames?», La Nouvelle Barre du Jour, $\mathrm{n}^{\text {os }}$ 110-111, février 1982, p. 91-97.

+ MORIN, Martin, «L'érotisme selon Jean-Louis Tripp», Planches, nº 11, été 2017, p. 19-29.

+ MORIN, Martin, "Les dessous de la création: Jean-Louis Tripp dans les coulisses d'Extases», Planches, $\mathrm{n}^{\circ}$ 12, automne 2017, p. 31-37.

+ MORIN, Martin, «Les dessous de la création: Tuer Vélasquez de Philippe Girard», Planches, $\mathrm{n}^{\circ} 13$, hiver 2017, p. 66-70.

+ OSSO, Didier, «Vagabondage: Jimmy Beaulieu», Pyro, n² 2, mars 2005, p. 16-23.

+ PELLETIER, Frédérique, «Ils sont fous ces Québécois», DBD. Les dossiers de la bande dessinée, $n^{\circ} 22$, avril 2004, p. 12-16.

+ PELLETIER, Frédérique, «En collant rouge et blanc», DBD. Les dossiers de la bande dessinée, $n^{\circ} 23$, juin 2004, p. 10-13. 
+ POIRIER, Raymond, «Créer autrement: sociofinancement et BD», Sentinelle, n 1, avril 2015, p. 23-31.

+ POMERLEAU, Luc, «La bande dessinée. Pourquoi le dessiner si on peut l'écrire?», Solaris, n 41, octobre 1981, p. 26-27.

+ POMERLEAU, Luc, «La bande dessinée. Le scénario et le découpage... », Solaris, n 42 , décembre 1981, p. 30-31.

+ POMERLEAU, Luc, "La bande dessinée de science-fiction et de fantastique au Québec», Protée, vol. X, n 2, été 1982, p. 83-86.

+ PYLYSER, Charlotte et Gabriel TREMBLAY-GAUDETTE, «The Visual Co-textualization of Spatio-Joual in the Motel Galactic Series», Christian Reyns et Gail de Vos (dir.), Alternatives francophones, vol. I, n 9, 2016, p. 189-211.

+ RABY, Georges, «Le printemps de la bande dessinée québécoise», Culture vivante, n 22 , septembre 1971, p. 12-23.

+ RANNOU, Maël, «La bande dessinée au Québec», Comix Club, n 10, janvier 2009, p. 71-87.

+ RANNOU, Maël, «Cartographier Montréal», Sentinelle, n², août 2015, p. 93-96.

+ ROBERT, Guy, «Dessin, caricature et bande dessinée», L'art au Québec depuis 1940, Montréal, La Presse, 1973, p. 206-219.

+ ROBERT, Vêronique, «Le parrain de la BD québécoise», Livre d'ici, vol. X, n 7, mars 1985, p. 8.

+ ROLFE, Christopher, «Round and About the Bande Dessinée Québécoise. From its Early Days to Chiendent», European Comic Art, vol. V, nº 1, printemps 2012, p. 9-24.

+ SAINT-DENIS, Félix, «Christian Quesnel: un monde de contrastes», Liaison, nº 108, septembre 2000, p. 6-9.

+ SAMSON, Jacques, «Une décennie de bandes dessinées québécoises», Imagine..., $\mathrm{n}^{\circ} 11$, hiver 1981, p. 9-20.

+ SAMSON, Jacques, «Il était une fois au Québec: Pierre Fournier et Réal Godbout», Les cahiers de la bande dessinée, $\mathrm{n}^{\circ}$ 72, novembre-décembre 1986, p. 42-44.

+ SAMSON, Jacques, «Panorama de la historieta en Quebec y en Canadá», Neuroptica, nº 5 , décembre 1988, p. 84-95.

+ SAMSON, Jacques, «Bande dessinée québécoise: sempiternels recommencements?», Réginald Hamel (dir.), Panorama de la littérature québécoise contemporaine, Montréal, Guérin, 1997, p. 282-307.

+ SAMSON, Jacques, «BD québécoise: sempiternels recommencements?», Trip, $\mathrm{n}^{\circ}$ 7, printemps 2012, p. 35-50.

+ SAMSON, Jacques, «L'étrange Monsieur Henriette Valium», Sentinelle, nº 1, avril 2015, p. 45-51.

+ SAOUTER CAYA, Catherine, «BD québécoise: une adolescence difficile», Nuit blanche, n 16 , décembre 1984-janvier 1985, p. 63-65.

+ SAOUTER CAYA, Catherine, "L'Eldorado et Iceberg: deux périodiques québécois de BD", Jacques Samson et André Carpentier (dir.), Actes. Premier Colloque de bande dessinée de Montréal, Montréal, Analogon, 1986, p. 30-41.

+ ST-CERNY GOSSELIN, Camille, «Mainmise, Québec-Presse et les revues de bande dessinée», Karim Larose et Frédéric Rondeau (dir.), La contre-culture au Québec, Montréal, Presses de l'Université de Montréal, coll. «Nouvelles études québécoises», 2016, p. 339-377.

+ ST-JACQUES, Marianne, «BDQ: cherchez la femme», Sentinelle, n 3, décembre 2015, p. 77-87.

+ TARDI, Valentin, «La BD québécoise existe!», Le Marginal, n 1, février 1996, p. 12-13. 
+ TEINTURIER, Sara, «L'Église et les bulles. Les représentations du catholicisme dans la bande dessinée francophone (années 1990-2010)», Études d'histoire religieuse, vol. LXXXII, nos 1-2, 2016, p. 57-74.

+ TESMOINGT, Daniel, «Portfolio Louis Lachance», L'Inédit, n 14, 2002, p. 40-50.

+ TESSIER, Marc, «Traquer l'Égo : la bande dessinée underground au Québec», Guillotine, vol. I, $\mathrm{n}^{\circ}$ 4, 1995, p. 1-8.

+ TESSIER, Marc, «The Montreal Comix Scene. When Solitudes Unite: A Personal History, 19872003», The Comics Journal, vol. V, 2005, p. 106-122.

+ THIBAULT, Gilles, «La bande dessinée au Québec», Médiart, nº 12 bis, décembre 1972, p. 2-9.

+ THIBAULT, Gilles, «La bande dessinée au Québec», Médiart, n 13, janvier 1973, p. 13-15.

+ THIBAULT, Gilles, «La bande dessinée au Québec», Yves Robillard (dir.), Québec Underground, 1962-1972, t. II, Montréal, Les éditions Médiart, 1973, p. 335-345.

+ TREMBLAY-GAUDETTE, Gabriel, «Morlac. Jouer à la lecture», Trip, nº 7, printemps 2012, p. 109-115.

+ TREMBLAY-GAUDETTE, Gabriel, «Du mauvais usage du terme "bédéesque" dans les médias québécois», Trip, nº 7, printemps 2012, p. 117.

+ TREMBLAY-GAUDETTE, Gabriel, «Dérives du moi dessiné, lecture de la bédé-réalité de Julie Delporte», Trip, nº 7, printemps 2012, p. 197.

+ TREMBLAY-GAUDETTE, Gabriel, «Écrire ce qui vient naturellement: la langue dans l’histoire de la bande dessinée québécoise», Aline Francœur (dir.), Adaptation dans les espaces francophones. Formes, expressions et diffusion, Presses de l'Université Laval, coll. «Culture française de l'Amérique», 2016, p. 112-138.

+ TURGEON, David, «Paratexte et bande dessinée», du9, avril 2008, en ligne: http://du9.org/ Paratexte-et-bande-dessinee (page consultée le 20 janvier 2018).

+ TURGEON, David, «Crise de l'autobiographie», du9, septembre 2010, en ligne: https://www. du9.org/dossier/crise-de-l-autobiographie/(page consultée le 20 janvier 2018).

+ VÉRONNEAU, Pierre, «Introduction à une lecture de la bande dessinée québécoise, 19401910», Stratégie. Lutte idéologique, nos 13-14, printemps 1976, p. 59-75.

+ VERREAULT-CÔTÉ, Carolane, «Vois-tu ce que je vois? L'habile mise en place d'une conscientisation socio-politique chez Guy Delisle», Trip, n ${ }^{\circ}$, printemps 2014, p.184-190.

+ VIAU, Michel, «Les aventures de Timothée, première bande dessinée à bulles de langue française», Le collectionneur de bandes dessinées, n 94, été 2001, p. 47.

+ VIAU, Michel, 100 ans de BD au Québec... et plus!, supplément du MensuHell, nº 54, Repentigny, Francis Hervieux éditeur, mai 2004, 20 p.

+ VIAU, Michel, «Hector Berthelot, le roi des journaux satiriques», La Semaine, vol. I, nº 48, 6 janvier 2006, p. 12-13 [cahier «Tout connaître»].

+ VIAU, Michel, «Grande presse et petits bonshommes. La naissance de la BDQ", Jimmy Beaulieu (dir.), Bears + Beer, Montréal, Mécanique générale, coll. «Formule Un», 2007, p. 15-51.

+ VIAU, Michel, «La BD au Québec: une route semée d'embûches», Québec français, nº 149, printemps 2008, p. 32-34.

+ VIAU, Michel, «Bédé, ciné, télé... La bande dessinée québécoise dans les autres médias», Trip, $\mathrm{n}^{\circ}$ 7, printemps 2012, p. 97-101.

+ VIAU, Michel, «De la grande presse à la librairie. Histoire de la bande dessinée québécoise», Collections, vol. I, nº 6, novembre 2014, p. 4-8.

+ VIAU, Michel, «L'année de la diversité. La production québécoise de bandes dessinées en $2014 »$, Sentinelle, n 1, avril 2015, p. 5-21. 
+ VIAU, Michel, «La filière québécoise. La BD franco-belge au Québec», Sentinelle, n² 2, août 2015, p. 7-29.

\section{I I. MÉMOIRES ET T HẼSES}

+ BEAULAC, Mario, BD, bandes dessinées québécoises et nouvelle culture (essai d'analyse sémiologique), mémoire de maîtrise, Montréal, Université de Montréal, 1974, 97 f.

+ BERTHIAUME, Jean-Michel, La métafiction en bande dessinée: métafiction dans les œuvres Bungalopolis de Jean-Paul Eid et Animal Man de Grant Morrison, mémoire de maîtrise, Montréal, Université Concordia, 2013, 137 f.

+ BOISVERT, Jacinthe, Albert Chartier, chroniqueur en bande dessinée d'un Québec en mutation, mémoire de maîtrise, Montréal, Université du Québec à Montréal, 1992, 175 f.

+ CAISSE, Sébastien, Le contrat d'édition de bandes dessinées, mémoire de maîtrise, Québec, Université Laval, 1994, 135 f.

+ DELPORTE, Julie, La bédé-réalité. La bande dessinée autobiographique à l'heure des technologies numériques, mémoire de maîtrise, Montréal, Université de Montréal, 2011, 113 f; [s. l.], Colosse, coll. «Colosse/Essais», [s. d.], 140 p.

+ FALARDEAU, Mira, L'humour visuel. Un modèle d'analyse visuelle des images comiques, mémoire de maîtrise, Québec, Université Laval, 1978, 124 f.

+ FALARDEAU, Mira, La bande dessinée faite par les femmes en France et au Québec depuis 1960, thèse de doctorat, Paris, Université Paris-Sorbonne/Institut des sciences de l'art, 1981, microfilms [Québec, Université Laval].

+ HARDY, Dominic, Drawn to Order. Henri Julien's Political Cartoons of 1899 and his career with Hugh Graham's Montreal Daily Star, 1888-1908, mémoire de maîtrise, Peterborough, Trent University, 1998, $274 \mathrm{f}$.

+ LAMOTHE, Stéphanie, Les modes d'expression du projet autobiographique dans la bande dessinée québécoise, mémoire de maîtrise, Montréal, Université du Québec à Montréal, 2011, $134 \mathrm{f}$.

+ LEMAY, Sylvain, Grotesque et satire dans les aventures de Michel Risque et de Red Ketchup de Réal Godbout et Pierre Fournier, mémoire de maîtrise, Montréal, Université du Québec à Montréal, 1996, $122 \mathrm{f}$.

+ LEMAY, Sylvain, Le "printemps» de la bande dessinée québécoise (1968-1975), thèse de doctorat, Montréal, Université du Québec à Montréal, 2010, 363 f.

+ LINDNER, Julien, "Joindre l'infiniment grand et l'infiniment québécois. » Le langage familier et populaire dans la bande dessinée québécoise, mémoire de maîtrise, Innsbruck, LeopoldFranzenz-Universität/Institut für Romanistik, 2015, 127 f.

+ OUELLET, Manon, Utilisation de la bande dessinée comme amorce d'une rééducation de la communication écrite. Étude de cas d'une élève de $\sigma^{e}$ année suivie en orthopédagogie, mémoire de maîtrise, Montréal, Université du Québec à Montréal, 1989, 360 f.

+ SAOUTER-CAYA, Catherine, La bande dessinée québécoise (1979 - 1984). Éléments pour une sémiologie de la bande dessinée, thèse de doctorat, Montréal, Université du Québec à Montréal, 1990, $337 \mathrm{f}$. 


\section{V. D O C U M ENTS PÉDAGOGIQUES}

+ BEAUREGARD, Jacques et Léandre TURCOTTE, Bande dessinée. Document pour l'enseignement du français au secondaire ( $2^{e}$ cycle), Montréal, Beauchemin, coll. «Balises», 1973, $40 \mathrm{p}$.

+ BERNARD, Lise, Bandes dessinées. Guide pédagogique et corrigé, illustrations de Daniel Dumont, Boucherville, Graficor, coll. «Les Scribouillards», 1988, 48 p.

+ BINAMÉ, Françoise et Ghislaine GODBOUT, Jeux linguistiques. Exploitation de la bande dessinée, Québec, ministère de l'Éducation, 1981, 6 f.

+ BLETON, Paul, Bande dessinée et figuration narrative. Cahier de l'étudiant, Sainte-Foy, TéléUniversité, 1992, 16 p.

+ BOUDREAU, Solange, Le temps dans la lecture d'une bande dessinée, Sainte-Foy/Montréal, PPMF-Laval/Éditions Ville-Marie, coll. «L'enseignement du français au primaire/Publications PPMF Laval», 1983, $92 \mathrm{p}$.

+ BOUSSER-BAL, Josèphe, Le français à l'élémentaire. L'intégration du matériel didactique à la pratique pédagogique, Rimouski, Université du Québec à Rimouski/GREME, coll. «Monographie. Université du Québec à Rimouski, Département des sciences de l'éducation/ PPMF», 1982, 248 p.

+ FALL, Khadiyatoulah et Hélène SAMSON, La bande dessinée: les éléments constitutifs suivi de La bande dessinée: production et lecture de récits, Chicoutimi, Université du Québec à Chicoutimi/PPMF, 1982, $49 \mathrm{f}$.

+ FARID, Georges, Exploitation pédagogique de la bande dessinée. Littérature enfantine (101), Montréal, Éditions Ville-Marie, coll. «Didactique du français au primaire/Publications PPMF», 1980, $113 \mathrm{p}$.

+ FARID, Georges et Caroline LEBLANC, Analyse expérimentale des «re-raconters» ou de l'interprétation de planches de bandes dessinées (B.D.) par les élèves de $6^{e}$ année au primaire. Rapport de l'avant-projet, Hull, Université du Québec à Hull, 1983, 135 p.

+ FARID, Georges, Code grammatical et pédagogique de la bande dessinée par l'exemple, Montréal, Agence d’Arc, 1989, 112 p. Précédemment publié sous le titre Matériel didactique pour l'apprentissage systématique et progressif du message verbo-iconique de la bande dessinée (1985).

+ GAGNON, Jean-Claude, Lire une bande dessinée: pratique, théorie, pratiques, Montréal, Éditions Ville-Marie, 1983, $217 \mathrm{p}$.

+ GUÉRETTE, Charlotte, Le roman (II) et la bande dessinée, Sainte-Foy, Université Laval, coll. «Littérature pour enfants», 1992, 43 p.

+ HOLT, William, Teaching literary concepts via the medium of comic strips, Montréal, Université McGill, 1974, 1 volume.

+ LANGLOIS, Richard, «Les étapes sommaires de réalisation d'une B.D.», Québec français, n 69, mars 1988, p. 39-42.

+ LAROCHELLE, Réal, La bande dessinée, Montréal, Hurtubise, coll. «En situation d'apprentissage», 1972, 38 p.

+ LEBRUN, Denis, «La bande dessinée. Initiation à un langage nouveau », Éducation Québec, nº, septembre-octobre 1977, p. 24-27.

+ ROBERGE, Hélène et Denyse BOURNEUF, La bande dessinée, $2^{\mathrm{e}}$ édition, Sainte-Foy/Montréal, PPMF-Laval/Éditions Ville-Marie, coll. «L'enseignement du français au primaire. Littérature enfantine/Publications PPMF Laval», 1980, 40 p. 
+ ROLLET, Gilles et Roger TREMBLAY, Parler et écrire avec la bande dessinée. Bloc pour 5 élèves, dessins de Effannob, Montréal, Centre éducatif et culturel, 1975, 60 f. Publié séparément en anglais sous le titre Speaking and Writing with Comic Strips: for 5 Students.

+ ROUX, Paul, La BD, l'art d'en faire. Méthode progressive pour apprendre à réaliser des bandes dessinées, de l'écriture du scénario à la planche en couleurs, Vanier, Centre franco-ontarien de ressources pédagogiques, $1994,80 \mathrm{p}$.

+ ROY, Pierre, Le goût de lire et la bande dessinée, Sherbrooke, Association canadienne pour l'avancement de la littérature de jeunesse, coll. «Lecture», 1991, 213 p.

+ THÉRIAULT, Lina et Richard LANGLOIS, Bande dessinée. Pour une didactique de l'expression et de la communication écrite au primaire, Sherbrooke, Université de Sherbrooke, 1980, 3 volumes (v. 1: Théorie, v. 2: Activités, v. 3: Expérimentations), 1155 p.

+ TRAN, Évelyne et Marie-José TRUDEL, La bande dessinée dans l'atelier d'écriture, Sainte-Foy/ Montréal, PPMF-Laval/Éditions Ville-Marie, coll. «L'enseignement du français au primaire/ Publications PPMF Laval», 1981, 59 p.

\section{DOCUMENTS AUDIO ET AUDIOVISUELS}

+ BLAQUIĖRE, Denis et Louis-Vincent BLAQUIÈRE (réal.), BDQC, saison 1, Montréal, Argus Films, ARTV, 2014.

+ BLAQUIĖRE, Denis et Louis-Vincent BLAQUIĒRE (réal.), BDQC saison 2, Montréal, Argus Films, ARTV, 2016.

+ DELPORTE, Julie (réal.) et Laurent BOUTIN, Dans ta bulle, CHOQ.ca, 2007-2015.

+ FORGET, Mathieu (réal.), Bédéphilement vôtre, CISM, 2011-2016.

+ LACROIX, Saël (réal.), Sur les traces d'Arthur, Montréal, Les films du 3 mars, 2016.

+ LEDUC, Jean-Dominic (réal.), Vous avez dit BD?, Productions Podcasse, 2017-2018.

+ POIRIER, Raymond (réal.), La vie en BD?, CKRL, 2011-2018. 